\title{
BUSINESS MODEL APROACH FOR QOE OPTIMIZED SERVICE DELIVERY
}

\author{
Jose I. Aznar, Eduardo Viruete, Julian Fernandez-Navajas, Jose Ruiz-Mas, \\ Jose Saldana and L. Casadesus \\ Communication Technologies Group (GTC), Aragon Institute of Engineering Research (I3A) \\ Dpt. IEC, Ada Byron Building, CPS, Univ. Zaragoza, 50018, Zaragoza, Spain \\ \{jiaznar, eviruete,navajas, jruiz, jsaldana, luis.casadesus\}@unizar.es
}

Keywords: $\quad$ QoE, B2B QoE model, End-user potential market.

\begin{abstract}
Current B2B (Business to Business) models are not capable to cover neither customer expectations in terms of quality nor personalization. Network, service and equipment providers are tied to traditional business models, missing the opportunity to increase their revenues derived from the integration of Quality of Experience (QoE) models in their frameworks. In this work, we propose a B2B QoE model which comprises the main guidelines to successfully integrate the QoE within the value chain and provide with added-value services to potential subscribers. We also evaluate the potential QoE end-user market for six European countries. Results indicate that there is a niche for QoE based models which rely on the joint action of value chain actors and its agreement with the regulatory environment.
\end{abstract}

\section{INTRODUCTION}

New multimedia services have acquired personalization and customization features which are enclosed under the concept of Quality of Experience (QoE). ITU-T has defined QoE as "the overall acceptability of an application or service, as perceived subjectively by the end-user, where the overall acceptability may be influenced by user expectations and context" (ITU-T, 2006).

The overall QoE demand entails a set of requirements which must be accomplished from technological and economical perspectives and in the context of the regulation action. In terms of technology, Equipment Providers (EPs), Service Providers (SPs) and Network Providers (NPs) are expected to handle much higher traffic levels, offering improved quality. From an economical perspective, the main difficulty hereby for providers is that the potential subscribers remain reluctant to pay more for QoE services. Also the uncertain attitude of National Regulation Authorities (NRAs) on QoE technologies might represent a showstopper as far as regulation actions do not clarify the New Regulation Framework (NRF).

From this situation, the question which arises is whether or not it exits a niche for the QoE, or alternatively, which are the key drivers that may lead value chain actors to include QoE B2B (Business to Business) models in their strategies to reach the customer?

The main issue of this work aims to address this question through the definition of a novel approach for a QoE B2B model. This model clearly advocates for driving innovation through alliances among operators and providers while keeping competitive. We also address the relation of the B2B model with the end-user market: there have been identified the key factors which may (positively or negatively) impact users' willingness to pay and QoE affinity.

The remainder of this paper is organized as follows: In section 2 the B2B model is explained. Section 3 presents the key factors which may impact B2C (Business to Consumer) relationships. User's affinity and willingness to pay are also tested. Section 4 concludes the paper.

\section{QOE B2B MODEL APPROACH}

\subsection{QoE B2B Model}

The QoE B2B model entails a shift on the side of markets to adopt new business roles. There are multiple individual markets which may be relevant 
for the B2B model definition. Nevertheless, all these markets can be combined to create a convergent environment with three global markets and their information flows located in two main levels: application service and network service levels (Figure 1). Market convergence increases the possibilities for services and content-related opportunities emerging for involved players.

Among the defined markets two possible options may take place to determine if they become or not strategic partners: "potential alliance" or "war".

The most important requirement that markets should achieve focuses on partnering strategies leading for a new revenue distribution model.

\subsection{Revenue Distribution Model}

High competitive business environments and falling prices in mature markets have favored the evolution of the operator model from a "walled garden" model to an "open garden" one.

In the "walled garden" model, services are directly retailed to end-users by operators and the number of content and service providers is limited by strict contractual agreements.

In contrast, in the "open garden" model, operators adopt a different approach, which consists of opening up their network capabilities (i.e. presence, location, identification, billing) to third parties, moving from a closed network model to a more suitable one, in which service rollouts can be faster to start obtaining revenues quickly.

All these factors may promote a greater interaction among business actors. Figure 2 schemes the different proposed relationships which enable to build a revenue flow map among the markets of the QoE value chain. Next section details the characteristics of these partnering strategies.

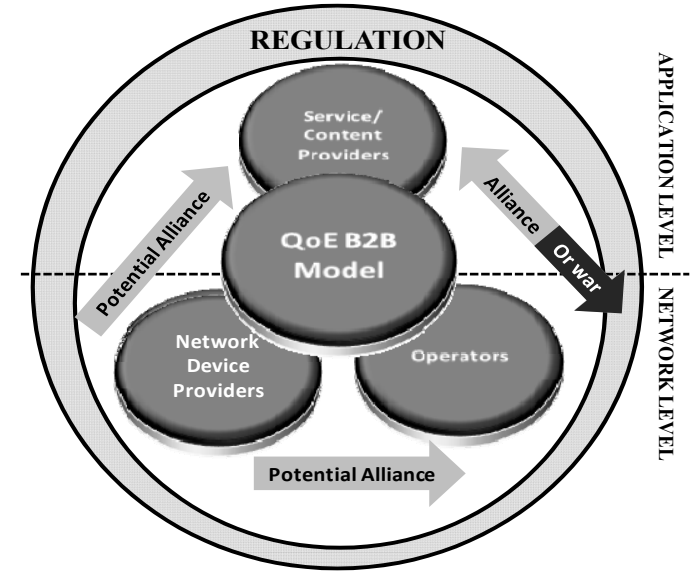

Figure 1: QoE business model based on market convergence.

\subsection{B2B Win-win Alliances}

\subsubsection{Operators and Equipment Providers (EPs)}

One possibility of revenue share can be identified between Telecom operators and EPs. EPs may deploy and manage QoE platforms, enabling operators to reduce CapEx and OpEx, in return of a percentage of the new revenue streams. The integration of $\mathrm{QoE}$ in operators' infrastructures entails the acquisition of new equipment to deploy QoE related enhancements. It is therefore necessary to establish relationships among EPs, chip industry and operators to provide QoE services.

\subsubsection{Telecom operators and Service and Content Providers (SPs/CPs)}

SPs and CPs have been largely keeping a reciprocal push and pull with operators based on an accentuated mistrust between them. On one hand, $\mathrm{CPs}$ and SPs are suspicious of enabling operators manage the content that is provided through their networks. On the other hand, operators still keep reluctant to guarantee QoE access levels to providers, without obtaining substantial profit from this. Thus, CPs and SPs have the opportunity to increase their revenues within the value chain if they shift to an open-mind perspective in the QoE market.

Besides, thanks to operators' network capabilities, SPs and CPs will be able to reach a larger number of customers, while ensuring high QoE.

\subsubsection{Alliances among Telecom Operators}

Competition in the Telecoms sector avoiding dominant market positions is a key driver for lower prices and substantially more attractive broadband speeds. Europe's digital deficit claims that the takeup rate of superfast BB (BroadBand) could be double in some European countries if networks were opened to competition. Moreover, the NRF should regulate BB service-packets fees across Europe.

\subsubsection{Advertisers also Take Part}

Users could remain reluctant to pay substantially more for the QoE enhanced services, an alreadyknown player might be integrated in the value chain: advertisers. Targeted advertisements could be included inside service applications and be optionally offered to users, subsidizing the service.

The QoE specific "personalization" feature is not limited to users' demanded contents, but can also be extended to the advertising industry. 


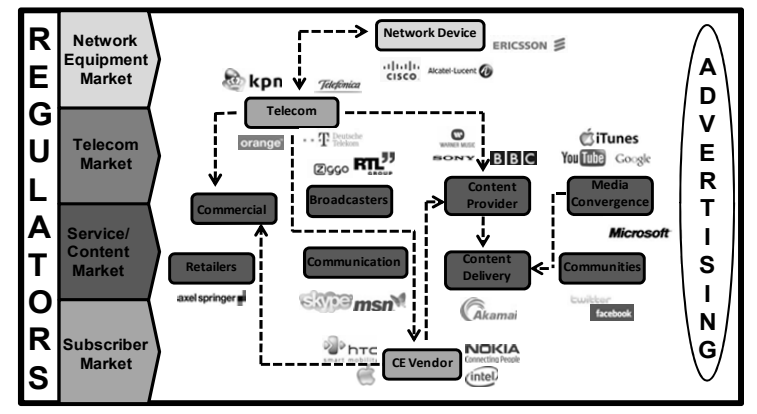

Figure 2: Revenue flow map among incumbent potential QoE markets.

\subsubsection{Regulation and the Global Market}

The telecom regulation action definitely impacts the market, since the laws and actions carried out by regulation authorities, set the basis for the B2B ecosystem map. The NRF relies into two main concepts: first, the need to ensure European customers to have a greater choice of BB SPs with prioritization, or differentiated products; and second, a clear focus on net neutrality. The NRF seems to match QoE expectances and guarantee the market deployment and evolution in the mid-term. However, both at the side of end-users and wholesalers have appeared opposite points of view.

\section{THE END-USER MARKET}

\subsection{Drivers/Showstoppers Factors which may Impact the B2C Model}

The success of the adoption of QoE to end-users depends on several factors which may positively or negatively impact their affinity and willingness to pay. We have identified three key factors covering the technological, social and legislatives fields.

From a technological perspective the current international broadband gap is the most important issue to be solved: Eastern and Southeast Europe's gap with its Western neighbors in terms of access to high-speed internet is widening and limiting the access to potential QoE subscribers. Concerning social trends, recent studies (Microsoft 2009), have forecasted during the last decade that Internet consumption would overtake TV during 2010. Nowadays, this is a fact. Finally, national regulation actions do not only consist of legislating on how net neutrality must be established in the B2B model, but also on setting the rules for subscribers' access to content. The main issue that CPs are currently experiencing deals with the legal piracy vacuum present in some countries, since it entails significant lower business perspectives than in countries where P2P sharing constitute an illegal activity.

\subsection{Study of End-user QoE Affinity}

We conclude this work with a quantitative approach of the end-user potential market. The approach enables to forecast whether or not the there is a will from the end-user side to acquire and pay for QoE services. We have addressed the end-user affinity analysis from a set of descriptors, for six European countries and also for the overall EU-27 former states.

The study has been conducted based on the measurement of the end-user potential market demand (EuPM) characterized by (1) taken and adapted from the marketing management environment (Kotler, 2006):

$$
\mathrm{EuPM}=\mathrm{N} \times \mathrm{v} \times \mathrm{Q} \times \mathrm{WP}
$$

Where the factor

$$
\mathrm{N} \times \mathrm{V}
$$

represents users' potential affinity in the QoE market. $Q$ is the acquired product quantity by a medium purchaser and WP represents the price assigned to the product of interest, in this case, the QoE. This study focuses on (2). $Q$ and $W P$ factors determination are out of the scope of this work.

$N$ represents the potential household BB fixedaccess penetration rate. $N$ values have been compiled from (TNS, 2010) and can be observed in Table 1. $v$ is the relevant percentage of $\mathrm{BB}$ households that may consume QoE services. This descriptor has been derived from two secondary descriptors: The "National Education Attainment" and the end-users" "age rank" descriptors. The main considerations for $v$ descriptor have been also categorized: assumptions related to the "age-rank" descriptor: people below 25 do not represent QoE potential consumers. People between 25 and 79 represent the most valuable group since they perceive a salary and are closely related too. People above 80 are neither technology nor QoE familiar.

The $v$ descriptor values (shown in Table 1) have been derived from public statistic data (UNESCO, 2010), (UNDP, 2009). The "National Education Attainment" descriptor has been divided into three levels: low, medium and high education attainment. 
Table 1: $N$ and $v$ values for end-user QoE affinity analysis.

\begin{tabular}{|l|c|c|c|}
\hline Country & $\begin{array}{c}\text { Number of } \\
\text { households } \\
\text { (Thousand) }\end{array}$ & $\begin{array}{c}\text { Household } \\
\text { BB } \\
\text { penetration } \\
\text { (\% of BB } \\
\text { households) }\end{array}$ & $\begin{array}{c}\text { Share of } \\
\text { relevant } \\
\text { QoE } \\
v(\% \text { of BB } \\
\text { households) }\end{array}$ \\
\hline Belgium & 4568 & $54 \%$ & $53.97 \%$ \\
\hline EU-27 & 202925.2 & $48 \%$ & $38 \%$ \\
\hline France & 27392.9 & $59 \%$ & $52.89 \%$ \\
\hline Germany & 39311.2 & $45 \%$ & $41 \%$ \\
\hline Netherlands & 7269.8 & $79 \%$ & $59.77 \%$ \\
\hline Spain & 17076.3 & $44 \%$ & $37.10 \%$ \\
\hline UK & 26753.3 & $58 \%$ & $57.07 \%$ \\
\hline
\end{tabular}

It has been assumed that low education attainment people do not represent QoE relevant consumers.

Figure 3 shows the relevant share of QoE enduser potential consumers $(N * v$ product) for each country. This represents an approach to determine the percentage of country population which, having access to QoE technology may be allied to the QoE market. Country values have been normalized to their corresponding total population.

The Netherlands and the UK present a major affinity for QoE consumption, whereas EU-27 and Spain values reflect a minor affinity. Results are directly impacted by the national BB penetration and the legal vacuum in terms of piracy (see section 3.1).

Two main affinity levels can be observed: On one side, there can be found countries in which $\mathrm{BB}$ deployment is over $50 \%$ (The Netherlands, Belgium, France and UK), where piracy and $\mathrm{P} 2 \mathrm{P}$ are forbidden (Germany and France) or both. These countries present an affinity degree over $25 \%$ of the total population. On the other level we find the EU27 and Spain, were the lower level affinity is caused by the lack of legislation in terms of P2P and the lower BB household penetration, which is still far from reaching top countries. Neither Spain nor the EU-27 reaches a 25\% of QoE affinity degree.

\section{CONCLUSIONS}

The QoE B2B market has been depicted as a landscape of alliances and partnerships among three main markets explained through the B2B QoE model. The revenue distribution model and the proposed alliances among SPs, EPs, CPs and Telecom Operators may drive the QoE integration and consist of a combination of personalized services and customized applications which attract end-users to the QoE market. Regulation authorities may accomplish a homogenized regulation to permit operators and providers low fares. The end-user potential affinity shows that they are not yet aware

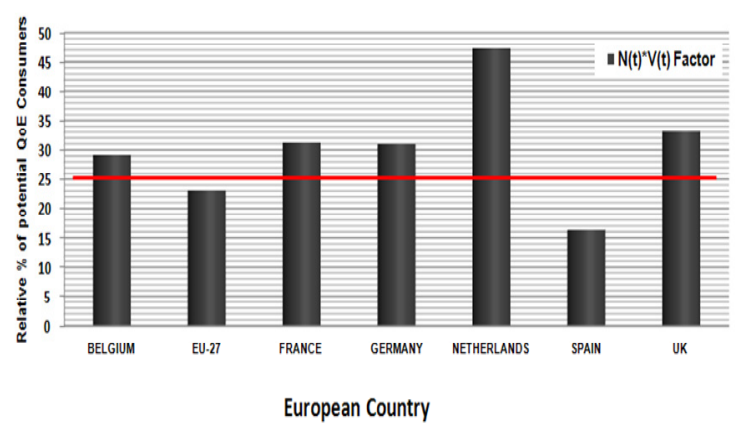

Figure 3: Relative country QoE affinity.

of the benefits that QoE based services provide. There is a demand, but it must be encouraged.

Summing up, the QoE market is hanging by a thread. There is a niche for the QoE market which mostly depends not only on how the BB is carried out, but also on the companies' marketing strategies.

\section{ACKNOWLEDGEMENTS}

This work has been partially financed by CPUFLIPI Project (MICINN TIN2010-17298) and the Catedra Telefonica of University of Zaragoza.

\section{REFERENCES}

ECTA. (2010). Europe's $€ 25$ bln digital deficit. ECTA press releases. Available at: < http://www.ectaportal. com/en/PRESS/ECTA-Press-Releases/2010/Europe-s25bln-digital-deficit $>$.

ITU-T (2007) ITU-T Recommendation P.10/G.100, Amendment1 (2007), Definition of QoE.

Kotler P., Keller K. (2006) Marketing Management. Prentice-Hall, 12 edition.

Microsoft. (2009). Europe Logs on. Available at: < http://www.scribd.com/doc/14065700/Europe-LogsOn-Microsoft-Study $>$.

TNS. (2010). E-Communications on Household Survey. Available at $:<$ http://ec.europa.eu/information_society /policy/ecomm/doc/library/ext_studies/household_10/r eport_en.pdf $>$.

UNDP. (2009). United Nations Development Program Human and Education report. Available at: $<$ http://hdrstats.undp.org/es/indicators/169.html $>$.

UNESCO. (2010). Global Education Digest 2010. Education Statistics Across the World. Available at: $<$ http://www.ifap.ru/library/book482.pdf $>$ 